\title{
Gauge-invariant truncation scheme for the Schwinger-Dyson equations of QCD
}

\author{
D. Binosi ${ }^{1, *}$ and J. Papavassiliou ${ }^{2, \dagger}$ \\ ${ }^{1}$ ECT* - European Centre for Theoretical Studies in Nuclear Physics and Related Areas, \\ Villa Tambosi, Strada delle Tabarelle 286, I-38050 Villazzano (TN), Italy. \\ ${ }^{2}$ Departamento de Física Teórica and IFIC, \\ Centro Mixto, Universidad de Valencia-CSIC, \\ E-46100, Burjassot, Valencia, Spain,
}

(Dated: December 17, 2007)

\begin{abstract}
We present a new truncation scheme for the Schwinger-Dyson equations of QCD that respects gauge invariance at any level of the dressed loop expansion. When applied to the gluon selfenergy, it allows for its non-perturbative treatment without compromising the transversality of the solution, even when entire sets of diagrams (most notably the ghost loops) are omitted, or treated perturbatively.
\end{abstract}

PACS numbers: 12.38.Aw, 12.38.Lg, 14.70.Dj 
Introduction - The quantitative understanding of the non-perturbative properties of Quantum Chromodynamics (QCD) [1] constitutes still one of the most challenging problems in particle physics. The basic building blocks of this theory are the Green's (correlation) functions of the fundamental degrees of freedom, gluons, quarks, and ghosts. Their non-perturbative structure is at the center stage of extensive research that could furnish invaluable clues for deciphering the infrared dynamics of QCD.

Lattice simulations are indispensable in this quest, since they capture all the nonperturbative information of the theory. It has become clear by now that the lattice simulations yield an infrared finite gluon propagator in the Landau gauge. This rather characteristic behavior has recently been firmly established using lattices with large volumes; in addition, the non-perturbative ghost propagator in the same gauge diverges, at a rate that deviates only mildly from the tree-level expectation [2]. These clean lattice results constitute a serious challenge for the QCD theorists: obtaining the same results from the theory formulated in the continuum is bound to expose a fundamental dynamical mechanism at work.

In the continuous formulation the dynamics of all Green's functions are determined by an infinite system of coupled non-linear integral equations known as Schwinger-Dyson equations (SDE) [3]. These equations are inherently non-perturbative and can be used to address problems related to e.g., chiral symmetry breaking, dynamical mass generation, and formation of bound states. Since this system involves an infinite hierarchy of equations, in practice one is severely limited in their use, and the need for a self-consistent truncation scheme is evident [4]. Devising such a scheme, however, is very challenging, especially in the context of non-abelian gauge theories, like QCD [5]. The central problem stems from the fact that the SDEs are built out of unphysical off-shell Green's functions; thus, the extraction of reliable physical information depends crucially on delicate all-order cancellations, which may be inadvertently distorted in the process of the truncation.

The situation may best exemplified with the SDE of the gluon propagator $\Delta_{\alpha \beta}(q)$. In the Feynman gauge,

$$
\Delta_{\alpha \beta}(q)=-i\left[\left(g_{\alpha \beta}-\frac{q_{\alpha} q_{\beta}}{q^{2}}\right) \Delta\left(q^{2}\right)+\frac{q_{\alpha} q_{\beta}}{q^{4}}\right],
$$

where $\Pi_{\alpha \beta}(q)=\left(g_{\alpha \beta}-q_{\alpha} q_{\beta} / q^{2}\right) \Pi\left(q^{2}\right)$ is the gluon self-energy and $\Delta^{-1}\left(q^{2}\right)=q^{2}+i \Pi\left(q^{2}\right)$. 
a)

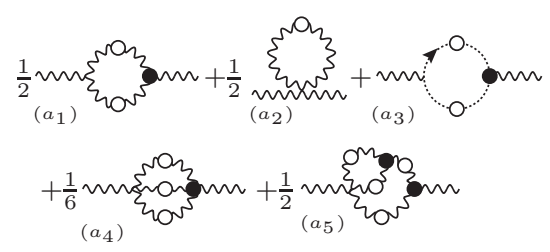

d)
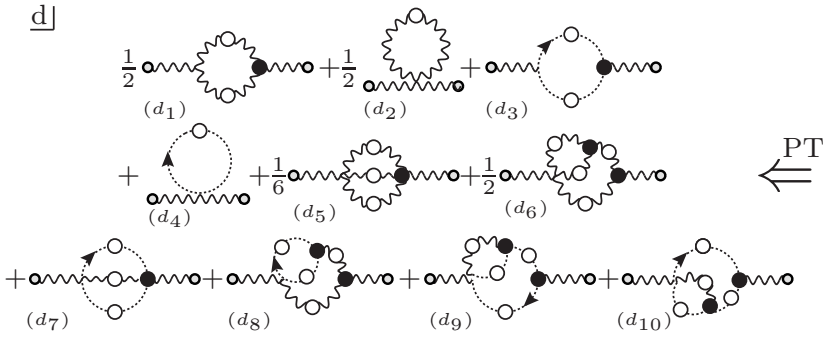

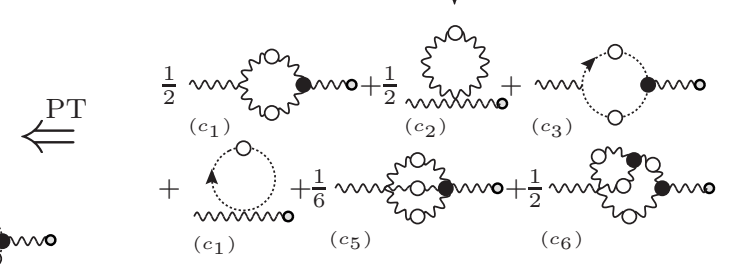

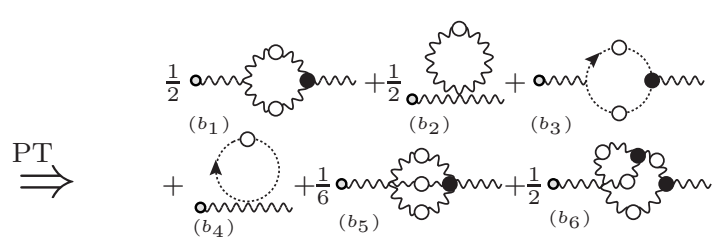

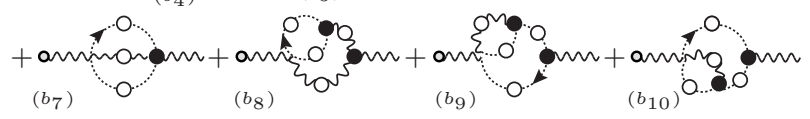

$\Downarrow \Gamma_{\widehat{A} A} \equiv \Gamma_{A \widehat{A}}$

$\lfloor c$

b

FIG. 1: The PT procedure to construct the new SDE of the gluon propagator. External legs ending in a gray circle represents background gluons. The corresponding Feynman rules can be found in [10].

The conventional SDE for $\Pi_{\mu \nu}$ reads

$$
\Pi_{\alpha \beta}(q)=\sum_{i=1}^{5}\left(a_{i}\right)_{\alpha \beta},
$$

where the diagrams $\left(a_{i}\right)$ are shown in Fig:1a. Since the self-energy enters in the latter diagrams (white blobs in the same figure), Eq.(2) constitutes a dynamical equation that can in principle determine $\Pi_{\alpha \beta}$. Due to general arguments based on the Becchi-Rouet-StoraTyutin (BRST) symmetry [6], $\Pi_{\alpha \beta}(q)$ is transverse, i.e. $q^{\alpha} \Pi_{\alpha \beta}(q)=0$. Notice, however, that enforcing this fundamental property on the rhs of Eq.(2), i.e., through the contraction of individual graphs by $q^{\alpha}$, is far from trivial, essentially due to the complicated Slavnov-Taylor identities (STI) satisfied by the fully-dressed vertices. As a result, the SDE of Fig 19a cannot be truncated without compromising the transversality of $\Pi_{\alpha \beta}(q)$. For example, keeping only graphs $\left(a_{1}\right)$ and $\left(a_{2}\right)$ is not correct even at one loop. Adding $\left(a_{3}\right)$ is still not sufficient for a SDE analysis, because (beyond one-loop) $q^{\alpha}\left[\left(a_{1}\right)+\left(a_{2}\right)+\left(a_{3}\right)\right]_{\alpha \beta} \neq 0$.

In this letter we present a new truncation scheme for the SDE of (quarkless) QCD that respects gauge invariance at any level of the dressed loop expansion. This becomes possible due to the drastic modifications implemented to the building blocks of the SD series, i.e. the off-shell Green's functions, following the field-theoretic method known as pinch tech- 
nique (PT) [7]. The PT is a well-defined algorithm that exploits systematically the BRST symmetry in order to construct new Green's functions endowed with very special properties. Most importantly, they satisfy abelian, Ward identities (WI) instead of the usual STIs, have correct analytic properties and displays only physical thresholds [8].

The PT rearrangement gives rise dynamically to a new SD series analogous to the one in Eq.(2), with the following characteristics: the graphs appearing on the rhs are made out of new vertices (Fig $1 \mathrm{l}$ ), but contain the conventional self-energy $\Pi_{\alpha \beta}$ as before. These new vertices correspond precisely to the Feynman rules of the Background Field Method (BFM) in the Feynman gauge, i.e., it is as if the external gluon had been converted dynamically into a background gluon. The lhs, in addition to the term $\Pi_{\alpha \beta}(q)$ already there, contains additional terms, also proportional to $\Pi_{\alpha \beta}(q)$, which are generated during the PT rearrangement of the original rhs of Eq.(2).

A new SD equation for the gluon propagator - The relevant PT rearrangements take place when the longitudinal momenta of the three-gluon vertex trigger the STIs satisfied by specific subsets of fully dressed vertices appearing in the ordinary perturbative expansion. Unlike QED, due to the non-linearity of the BRST transformations, these STIs are realized through auxiliary (ghost) Green's functions involving composite operators such as $\langle 0| T[s \Phi(x) \cdots|0\rangle$, where $s$ is the BRST operator and $\Phi$ is a generic QCD field. It turns out that the most efficient framework for dealing with these type of objects is the so-called Batalin-Vilkovisky formalism [12]. In this framework, one adds to the original gauge-invariant Lagrangian $\mathcal{L}_{\mathrm{I}}$ the term $\mathcal{L}_{\mathrm{BRST}}=\sum_{\Phi} \Phi^{*} s \Phi$, coupling the composite operators $s \Phi$ to the BRST invariant external sources (usually called anti-fields) $\Phi^{*}$, to obtain the new Lagrangian $\mathcal{L}_{\mathrm{BV}}=\mathcal{L}_{\mathrm{I}}+$ $\mathcal{L}_{\text {BRST. }}$. One advantage of this formulation is that it allows one to express the STIs of the theory in terms of auxiliary functions which can be constructed using a well-defined set of Feynman rules (derived from $\mathcal{L}_{\mathrm{BRST}}$ ). In particular, the usual STI satisfied by the three-gluon vertex, an essential ingredient in the ensuing construction, assumes the form

$q^{\alpha} \Gamma_{A_{\alpha}^{a} A_{\mu}^{m} A_{\nu}^{n}}\left(k_{1}, k_{2}\right)=q^{2} D^{a a^{\prime}}(q)\left[\Gamma_{c^{a^{\prime}} A_{\nu}^{n} A_{d}^{* \gamma}}\left(k_{2}, k_{1}\right) \Gamma_{A_{\gamma}^{d} A_{\mu}^{m}}\left(k_{1}\right) \Gamma_{c^{a^{\prime}} A_{\mu}^{m} A_{d}^{* \gamma}}\left(k_{1}, k_{2}\right) \Gamma_{A_{\gamma}^{d} A_{\nu}^{n}}\left(k_{2}\right)\right]$,

where $\Gamma_{A_{\alpha}^{a} A_{\beta}^{b}}(q)=\left(\Delta^{-1}\right)_{\alpha \beta}^{a b}(q)-i \delta^{a b} q_{\alpha} q_{\beta}\left[\right.$ with $\left.-\Gamma_{A_{\alpha} A_{\beta}}(q)=\Pi_{\alpha \beta}(q)\right]$, and the auxiliary function $\Gamma_{c A A^{*}}$, given in Fig, 2 a, is nothing but the standard function appearing in the conventional derivation [13] now written in the anti-field language. An important property of auxiliary functions involving the gluon anti-field, $A^{*}$, is encoded into the so-called Faddeev- 

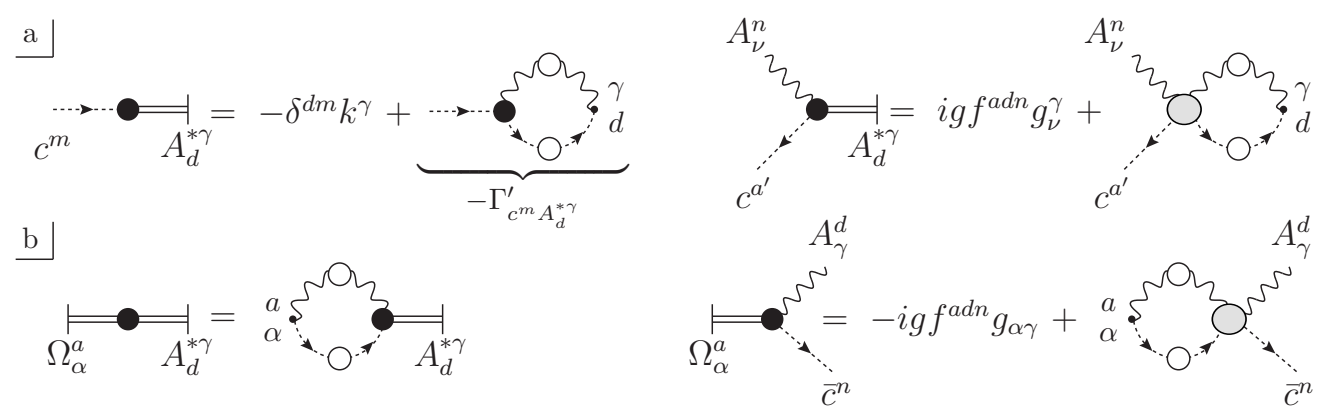

FIG. 2: The auxiliary functions $-\Gamma_{c^{m} A_{d}^{* \gamma}},-\Gamma_{\Omega_{\alpha}^{a} A_{d}^{* \gamma}}, i \Gamma_{c^{a^{\prime}} A_{\nu}^{n} A_{d}^{* \gamma}}$ and $i \Gamma_{\Omega_{\alpha}^{a} A_{\gamma}^{d} \bar{c}^{n}}$. Black and white blobs represent one-particle irreducible and connected Green's functions, respectively, while gray blobs are connected kernels.

Popov equation: $\frac{\delta \Gamma}{\delta \bar{c}^{a}}+i q^{\mu} \frac{\delta \Gamma}{\delta A_{\mu}^{* a}}=0$. This equation amounts to the simple statement that contracting $A^{*}$ with its own momentum $q$ converts it to an anti-ghost, $\bar{c}$. This property will be used extensively in what follows. In addition, one can obtain a set of useful identities relating Green's functions of background fields to those of quantum fields. These Background Quantum Identities (BQIs) [11] are realized through auxiliary functions involving normal fields, anti-fields, and a background source $\Omega$, coupled through the term $-g f^{a m n} \bar{c}^{a} \Omega_{\mu}^{m} A_{n}^{\mu}$, see Fig 2b. The BQIs satisfied by the gluon propagator are

$$
\begin{aligned}
& i \Gamma_{\widehat{A}_{\alpha}^{a} A_{\beta}^{b}}(q)=\left[i g_{\alpha}^{\gamma} \delta^{a d}+\Gamma_{\Omega_{\alpha}^{a} A_{d}^{* \gamma}}(q)\right] \Gamma_{A_{\gamma}^{d} A_{\beta}^{b}}(q), \\
& i \Gamma_{\widehat{A}_{\alpha}^{a} \widehat{A}_{\beta}^{b}}(q)=\left[i g_{\alpha}^{\gamma} \delta^{a d}+\Gamma_{\Omega_{\alpha}^{a} A_{d}^{* \gamma}}(q)\right] \Gamma_{A_{\gamma}^{d} \widehat{A}_{\beta}^{b}}(q),
\end{aligned}
$$

which can be combined into the single identity

$$
\begin{aligned}
i \Gamma_{\widehat{A}_{\alpha}^{a} \widehat{A}_{\beta}^{b}} & =i \Gamma_{A_{\alpha}^{a} A_{\beta}^{b}}+\Gamma_{\Omega_{\alpha}^{a} A_{d}^{* \gamma}} \Gamma_{A_{\gamma}^{d} A_{\beta}^{b}}+\Gamma_{\Omega_{\beta}^{b} A_{d}^{* \gamma}} \Gamma_{A_{\alpha}^{a} A_{\gamma}^{d}} \\
& +\Gamma_{\Omega_{\alpha}^{a} A_{d}^{* \gamma}} \Gamma_{A_{\gamma}^{d} A_{\epsilon}^{e}} \Gamma_{\Omega_{\beta}^{b} A_{e}^{* \epsilon}} .
\end{aligned}
$$

Other BQIs needed in our construction will be

$$
\begin{aligned}
i \Gamma_{\widehat{A}_{\alpha}^{a} \varphi \phi}\left(k_{1}, k_{2}\right) & =\left[i g_{\alpha}^{\gamma} \delta^{a d}+\Gamma_{\Omega_{\alpha}^{a} A_{d}^{* \gamma}}(q)\right] \Gamma_{\varphi A_{\gamma}^{d} \phi}\left(k_{1}, k_{2}\right) \\
& +R_{\Omega_{\alpha}^{a} \varphi \phi}\left(k_{1}, k_{2}\right)
\end{aligned}
$$

where $(\varphi, \phi) \in\left\{(A, A),(c, \bar{c}),\left(c, A^{*}\right)\right\}$, and

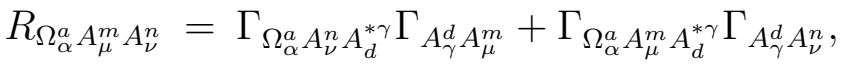

$$
\begin{aligned}
& R_{\Omega_{\alpha}^{a} c^{m} \bar{c}^{n}}=-\Gamma_{c^{m} A_{d}^{* \gamma}} \Gamma_{\Omega_{\alpha}^{a} A_{\gamma}^{d} \bar{c}^{n}}-\Gamma_{\Omega_{\mu}^{a} c^{m} c^{* d}} \Gamma_{c^{d} \bar{c}^{n}}, \\
& R_{\Omega_{\alpha}^{a} c^{m} A_{\nu}^{* n}}=-\Gamma_{c^{m}} A_{d}^{* \gamma} \Gamma_{\Omega_{\alpha}^{a} A_{\gamma}^{d} A_{\nu}^{* n}}-\Gamma_{\Omega_{\alpha}^{a} c^{m} c^{* d}} \Gamma_{c^{d} A_{\nu}^{* n}} .
\end{aligned}
$$


Equipped with these relations we may now proceed to the derivation of our main result. The aim will be to start from the conventional SDE of Fig 1a and generate dynamically through the PT algorithm all the terms appearing in the BQI of Eq.([6), thus arriving at the SDE equation of Fig.11. This will be accomplished by constructing the two BQIs of Eq.(4) and (5), one at a time. The starting point is diagram $\left(a_{1}\right)$ of Fig,1a. The tree-level three gluon vertex $\Gamma$ can be decomposed [7] into the sum $\Gamma^{\mathrm{F}}+\Gamma^{\mathrm{P}}$, where (factoring out the color structure)

$$
\begin{aligned}
& i \Gamma_{A_{\alpha} A_{\mu} A_{\nu}}^{\mathrm{F}}\left(k_{1}, k_{2}\right)=g_{\mu \nu}\left(k_{1}-k_{2}\right)_{\alpha}-2 q_{\mu} g_{\alpha \nu}+2 q_{\nu} g_{\alpha \mu}, \\
& i \Gamma_{A_{\alpha} A_{\mu} A_{\nu}}^{\mathrm{P}}\left(k_{1}, k_{2}\right)=g_{\alpha \nu} k_{1 \mu}-g_{\alpha \mu} k_{2 \nu} .
\end{aligned}
$$

This splitting assigns a special role to the physical momentum $q$, making $\Gamma^{\mathrm{F}}$ Bose symmetric only with respect to the $A_{\mu}$ and $A_{\nu}$ legs inside the loop. In fact, $\Gamma^{\mathrm{F}}$ coincides with the BFM vertex $\Gamma_{\widehat{A}_{\alpha} A_{\mu} A_{\nu}}^{(0)} \cdot \Gamma^{\mathrm{P}}$ contains the longitudinal momenta that will get contracted with the full three-gluon vertex, triggering the STI of Eq.(3). The result will be $\left(a_{1}\right)=\left(a_{1}\right)^{\mathrm{F}}+\left(a_{1}\right)^{\mathrm{P}}$, with $\left(a_{1}\right)^{\mathrm{F}}$ coinciding with diagram $\left(b_{1}\right)$, and

$$
\begin{aligned}
& \left(a_{1}^{\mathrm{P}}\right)_{\alpha \beta}^{a b}=-i \Gamma_{\Omega_{\alpha}^{a} A_{d}^{* \gamma}}(q) \Gamma_{A_{\gamma}^{d} A_{\beta}^{b}}(q)-i g f^{a m d}\left\{\int_{k_{1}} k_{2 \alpha} D\left(k_{1}\right) D\left(k_{2}\right) \Gamma_{c^{m}} A_{\beta}^{b} \bar{c}^{d}\left(-q, k_{2}\right)\right. \\
& \left.+\int_{k_{1}} D\left(k_{1}\right) \Gamma_{c^{e} A_{\alpha}^{* d}}^{\prime}\left(k_{2}\right) D\left(k_{2}\right) \Gamma_{c^{m} A_{\beta}^{b} \bar{c}^{e}}\left(-q, k_{2}\right)+i \int_{k_{1}} D\left(k_{1}\right) \Gamma_{c^{m} A_{\beta}^{b} A_{\alpha}^{* d}}\left(-q, k_{2}\right)\right\} .
\end{aligned}
$$

In the equation above we have used the ghost $\operatorname{SDE} k^{2} D(k)=1-i \Gamma_{c \bar{c}}(k) D(k)$ to transform a tree-level ghost propagator appearing in the second term of the rhs into a full one. The first integral on the rhs of Eq.(10) symmetrizes the ghost-gluon vertex of $\left(a_{3}\right)$, giving rise to the characteristic BFM vertex $\propto\left(k_{1}-k_{2}\right)_{\alpha}$, and thus to diagram $\left(b_{3}\right)$. The second term coincides precisely with the diagram $\left(b_{10}\right)$; the third term (see Fig,2) gives rise to diagram $\left(b_{4}\right)$ [through the tree-level part of $\Gamma_{c A A^{*}}$, as well as $\left(b_{6}\right),\left(b_{7}\right)$ and $\left(b_{8}\right)$. Finally, due to the fact that the four gluon vertices $\Gamma_{\widehat{A} A A A}$ and $\Gamma_{A A A A}$ coincide at tree-level, we will have $\left(a_{2}\right)=\left(b_{2}\right),\left(a_{4}\right)=\left(b_{5}\right)$, and $\left(a_{5}\right)=\left(b_{6}\right)$. Thus taking into account the first term in Eq.(10) we have dynamically reproduced the propagator BQI of Eq.(4).

At this point we have constructed $\Gamma_{\widehat{A}_{\alpha}^{a} A_{\beta}^{b}}(q)$; the next step will be to exploit the obvious equality $\Gamma_{\widehat{A}_{\alpha}^{a} A_{\beta}^{b}}(q)=\Gamma_{A_{\alpha}^{a} \widehat{A}_{\beta}^{b}}(q)$ to interchange the background and quantum legs (see Fig.1 $\mathrm{k}$ ). This introduces a considerable simplification: on the one hand we keep identifying the pinching momenta from the the PT decomposition of the (tree-level) $\Gamma$, while on the other hand the equality between diagrams $\left(c_{5}\right),\left(c_{6}\right)$ and $\left(d_{5}\right),\left(d_{6}\right)$ is immediate. 
Let us now carry out the PT splitting of Eq.(9) to diagram $\left(c_{1}\right)$. The $\Gamma^{\mathrm{F}}$ part of the vertex generates directly diagram $\left(d_{1}\right)$; the longitudinal momenta contained in $\Gamma^{\mathrm{P}}$ get contracted as before with the full three-gluon vertex, which, however, has now an external background leg. Using Eq.(7) with $(\varphi, \phi)=(A, A)$, we get

$$
\left(c_{1}^{\mathrm{P}}\right)_{\alpha \beta}^{a b}=-i\left[i g_{\beta}^{\gamma} \delta^{b d}+\Gamma_{\Omega_{\beta}^{b} A_{d}^{* \gamma}}(q)\right]\left(a_{1}^{\mathrm{P}}\right)_{\alpha \gamma}^{a d}+g f^{a m n} \int_{k_{1}} \Delta_{\alpha}^{\nu}\left(k_{2}\right) \frac{k_{1}^{\mu}}{k_{1}^{2}} R_{\Omega_{\beta}^{b} A_{\mu}^{m} A_{\nu}^{n}}\left(k_{1}, k_{2}\right) .
$$

The presence of the prefactor $i g_{\beta}^{\gamma} \delta^{b d}+\Gamma_{\Omega_{\beta}^{b}} A_{d}^{* \gamma}$ allows one to use the BQIs of Eq.(7) to convert the full vertices $\Gamma_{c A \bar{c}}$ and $\Gamma_{c A A^{*}}$, appearing in the last three terms of $\left(a_{1}^{\mathrm{P}}\right)$, into $\Gamma_{c \widehat{A} \bar{c}}$ and $\Gamma_{c \widehat{A} A^{*}}$, respectively. This operation has two effects: $(i)$ it generates $\left(d_{7}\right),\left(d_{8}\right),\left(d_{9}\right)$, and $\left(d_{10}\right)$, plus the contribution needed to convert $\left(c_{3}\right)$ and $\left(c_{4}\right)$ into $\left(d_{3}\right)$ and $\left(d_{4}\right)$, respectively; (ii) it gives rise to leftover contributions given by the three integrals appearing in Eq.(10) where the corresponding vertex is replaced by either $-R_{\Omega c \bar{c}}$ or $-R_{\Omega c A^{*}}$. These latter terms cancel exactly against the second term in Eq.(11), after its tree-level contribution has been extracted and used to convert $\left(c_{2}\right)$ into $\left(d_{2}\right)$. At this point we have generated all diagrams of Fig.1 [14]. In addition, using the BQI of Eq.(44) (already proven in the previous step), the term in $\left(a_{1}^{\mathrm{P}}\right)$ proportional to $\Gamma_{\Omega A^{*}}$ will give precisely $-i \Gamma_{\Omega_{\alpha}^{a} A_{d}^{* \gamma}}(q) \Gamma_{A_{\gamma}^{d} \widehat{A}_{\beta}^{b}}(q)$. Thus, we have constructed the full BQI of Eq.(5). Having dynamically realized the BQIs of Eqs.(4) and (5), we can combine them into Eq.(6), which constitutes the announced result. We emphasize that $(i)$ all rearrangements have been induced by the PT manipulation of only one diagram [ $\left(a_{1}\right)$ and $\left(c_{1}\right)$ of Fig,1] and (ii) all quantities encountered exist in the conventional formulation. In that sense, the Batalin-Vilkovisky formalism serves simply as an efficient way of keeping track of them.

Discussion - The new SD series just constructed reads

$$
\left[1+G\left(q^{2}\right)\right]^{2} \Pi_{\alpha \beta}(q)=\sum_{i=1}^{10}\left(d_{i}\right)_{\alpha \beta}
$$

where $G$ is defined as the part of $\Gamma_{\Omega_{\alpha} A_{\beta}^{*}}$ proportional to $g_{\alpha \beta}: \Gamma_{\Omega_{\alpha} A_{\beta}^{*}}(q)=i G\left(q^{2}\right) g_{\alpha \beta}+\sim q_{\alpha} q_{\beta}$. The rhs of Eq.(12) has a very special structure. The diagrams of Fig.1d can be separated into four subgroups $\left[\left(d_{1}\right),\left(d_{2}\right)\right],\left[\left(d_{3}\right),\left(d_{4}\right)\right],\left[\left(d_{5}\right),\left(d_{6}\right)\right]$, and $\left[\left(d_{7}\right),\left(d_{8}\right),\left(d_{9}\right),\left(d_{10}\right)\right]$, corresponding to one- or two-loop dressed gluonic or ghost contributions. Due to the abelian WIs satisfied by these new vertices, the contribution of each of the four subgroups is individually transverse [15]. 
The practical implications of this property for the treatment of the SD series are farreaching, since it furnishes a systematic, manifestly gauge-invariant truncation scheme. In the case of the gluon self-energy, for instance, the transversality of the answer is guaranteed at every step. Specifically, keeping only the diagrams in the first group, we obtain the truncated SDE

$$
\Pi_{\alpha \beta}(q)=\left[1+G\left(q^{2}\right)\right]^{-2}\left[\left(d_{1}\right)+\left(d_{2}\right)\right]_{\alpha \beta},
$$

and we have that $q^{\alpha}\left[\left(d_{1}\right)+\left(d_{2}\right)\right]_{\alpha \beta}=0$ by virtue of $q^{\alpha} \Gamma_{\widehat{A}_{\alpha}^{a} A_{\mu}^{m} A_{\nu}^{n}}\left(k_{1}, k_{2}\right)=g f^{a m n}\left[\Delta_{\mu \nu}^{-1}\left(k_{1}\right)-\right.$ $\left.\Delta_{\mu \nu}^{-1}\left(k_{2}\right)\right]$. Therefore, $\Pi_{\alpha \beta}(q)$ is transverse, as it should, despite the omission of the remaining graphs (most notably the ghost loops). In fact, one can envisage the possibility of employing completely different treatments for each subgroup: for example, one may treat the graphs $\left(d_{1}\right)$ and $\left(d_{2}\right)$ non-perturbatively, while opting for a perturbative treatment of the ghost diagrams $\left(d_{3}\right)$ and $\left(d_{4}\right)$, without compromising the transversality of the self-energy. The price one has to pay is the need to consider the additional SDE governing $G$ (see Fig,2b). Notice, however, that the approximations employed for the treatment of this latter SDE will not interfere with the transversality of $\Pi_{\alpha \beta}$. The abelian WIs furnish an additional technical advantage: one may use gauge-technique inspired Ansätze, a common practice when dealing with the SDE of QED [4], to express the vertices in terms of propagators, in such a way as to automatically enforce gauge invariance. Finally, notice that $(i)$ the SDEs for the QCD vertices can be constructed in a very similar way [16], and (ii) the analysis presented here can be generalized to other gauges (e.g., the Landau gauge) using the methodology developed in $[9]$.

In conclusion, the new SD series constructed in this letter provides a powerful tool for the systematic exploration of the non-perturbative sector of QCD, allowing the study of the fundamental Green's functions in a manifestly gauge-invariant way.

Acknowledgments: DB thanks the Physics Department of the University of Valencia, where part of this work has been carried out. JP is supported by the MEC grant FPA 2005-01678 and the Fundación General of the UV. Diagrams drawn using JaxoDraw [17].

* Electronic address: binosi@ect.it

† Electronic address: joannis.papavassiliou@uv.es 
[1] W. J. Marciano and H. Pagels, Phys. Rept. 36, 137 (1978).

[2] I. L. Bogolubsky, E. M. Ilgenfritz, M. Muller-Preussker and A. Sternbeck, arXiv:0710.1968 [hep-lat]; A. Cucchieri and T. Mendes, arXiv:0710.0412 [hep-lat]; P. O. Bowman et al., arXiv:hep-lat/0703022.

[3] F. J. Dyson, Phys. Rev. 75, 1736 (1949); J. S. Schwinger, Proc. Nat. Acad. Sci. 37, 452 (1951); Proc. Nat. Acad. Sci. 37, 455 (1951).

[4] D. C. Curtis and M. R. Pennington, Phys. Rev. D 42, 4165 (1990); Phys. Rev. D 48, 4933 (1993);

[5] S. Mandelstam, Phys. Rev. D 20, 3223 (1979).

[6] C. Becchi, A. Rouet and R. Stora, Annals Phys. 98, 287 (1976); I. V. Tyutin, Lebedev Institute Report, 75-39.

[7] J. M. Cornwall, Phys. Rev. D 26, 1453 (1982); J. M. Cornwall and J. Papavassiliou, Phys. Rev. D 40, 3474 (1989).

[8] J. Papavassiliou and A. Pilaftsis, Phys. Rev. Lett. 75, 3060 (1995); Phys. Rev. D 53, 2128 (1996); Phys. Rev. D 54, 5315 (1996); Phys. Rev. Lett. 80, 2785 (1998); Phys. Rev. D 58, $053002(1998)$.

[9] A. Pilaftsis, Nucl. Phys. B 487, 467 (1997).

[10] The BFM preserves the symmetry of the action under ordinary gauge transformations with respect to the background gauge field $\widehat{A}_{\mu}^{a}$, while the quantum $A_{\mu}^{a}$ ( appearing in loops ) transforms homogeneously. As a result, the background $n$-point functions satisfy QED-like WIs. See L. F. Abbott, Nucl. Phys. B 185, 189 (1981).

[11] D. Binosi and J. Papavassiliou, Phys. Rev. D 66, 025024 (2002); Phys. Rev. D 66, 111901 (2002); J. Phys. G 30, 203 (2004); JHEP 0703, 041 (2007).

[12] I. A. Batalin and G. A. Vilkovisky, Phys. Rev. D 28, 2567 (1983); Phys. Lett. B 69, 309 (1977).

[13] J. S. Ball and T. W. Chiu, Phys. Rev. D 22, 2550 (1980).

[14] The SDE for $\widehat{\Delta}$ has been derived within the BFM by R. B. Sohn, Nucl. Phys. B 273, 468 (1986) and A. Hadicke, JENA-N-88-19.

[15] A. C. Aguilar and J. Papavassiliou, JHEP 0612, 012 (2006).

[16] D. Binosi and J. Papavassiliou, in preparation.

[17] D. Binosi and L. Theussl, Comput. Phys. Commun. 161, 76 (2004). 